\title{
Variable utility of mosaic attenuation to distinguish fibrotic hypersensitivity pneumonitis from idiopathic pulmonary fibrosis
}

\author{
Joseph Barnett (1) ${ }^{1}$, Philip L. Molyneaux², Bhavin Rawal', Rezaur Abdullah³, \\ Samanjit S. Hare ${ }^{4}$, Rama Vancheeswaran ${ }^{3}$, Sujal R. Desai ${ }^{1}$, Toby M. Maher ${ }^{2}$, \\ Athol U. Wells ${ }^{5}$ and Anand Devaraj ${ }^{1,2}$
}

Affiliations: ${ }^{1}$ Dept of Radiology, Royal Brompton Hospital, London, UK. ${ }^{2}$ National Heart and Lung Institute, Imperial College London, London, UK. ${ }^{3}$ Dept of Respiratory Medicine, Barnet Hospital, Royal Free NHS Foundation Trust, London, UK. ${ }^{4}$ Dept of Radiology, Barnet Hospital, Royal Free NHS Foundation Trust, Royal Free NHS Foundation Trust, London, UK. ${ }^{5}$ Interstitial Lung Disease Unit, Royal Brompton Hospital, London, UK.

Correspondence: Joseph Barnett, Dept of Radiology, Royal Brompton Hospital, Sydney Street, London, SW3 2NP, UK. E-mail: j.barnett2arbht.nhs.uk

@ERSpublications

The headcheese sign is the form of mosaic attenuation most specific for distinguishing fibrotic hypersensitivity pneumonitis from idiopathic pulmonary fibrosis http://bit.ly/2DxqQKs

Cite this article as: Barnett J, Molyneaux PL, Rawal B, et al. Variable utility of mosaic attenuation to distinguish fibrotic hypersensitivity pneumonitis from idiopathic pulmonary fibrosis. Eur Respir J 2019; 54: 1900531 [https://doi.org/10.1183/13993003.00531-2019].

\section{ABSTRACT}

Background: Mosaic attenuation on computed tomography (CT) has been identified in international guidelines as an important diagnostic feature of fibrotic hypersensitivity pneumonitis (FHP) as opposed to idiopathic pulmonary fibrosis (IPF). However, mosaic attenuation comprises several different radiological signs (low-density lobules, preserved lobules, air trapping and the so-called "headcheese sign") which may have differing diagnostic utility. Furthermore, the extent of mosaic attenuation required to distinguish these two diagnoses is uncertain and thresholds of mosaic attenuation from international guidelines have not been validated.

Methods: Inspiratory and expiratory CT scans were evaluated by two readers in 102 patients (IPF $n=57$; FHP $n=45$ ) using a semiquantitative scoring system for mosaic attenuation. Findings were validated in an external cohort from a secondary referral institution (IPF $n=34$; FHP $n=28$ ).

Results: Low-density lobules and air trapping were a frequent finding in IPF, present in up to $51 \%$ of patients. A requirement for increasing extent of low-density lobules and air trapping based on guidelines (American Thoracic Society and Fleischner Society) was associated with increased specificity for the diagnosis of FHP (0.96 and 0.98 , respectively) but reduced sensitivity ( 0.16 and 0.20 , respectively). The headcheese sign was found to be highly specific (0.93) and moderately sensitive (0.49) for a highconfidence diagnosis of FHP. The high specificity of the headcheese sign was maintained in the validation cohort and when patients with other CT features of FHP were excluded.

Conclusion: Mosaic attenuation is a frequent finding in IPF. However, the headcheese sign can be confidently considered as being inconsistent with a diagnosis of IPF and specific for FHP. 


\section{Introduction}

A common dilemma in the diagnosis of fibrosing lung disease is distinguishing idiopathic pulmonary fibrosis (IPF) from fibrotic hypersensitivity pneumonitis (FHP). This distinction is important because these two conditions have different treatments and outcomes.

Several studies have identified the presence of mosaic attenuation and air trapping on computed tomography $(\mathrm{CT})$ as being important in the radiological diagnosis of hypersensitivity pneumonitis $[1,2]$ and FHP [3]. This is reflected in the 2011 American Thoracic Society (ATS)/European Respiratory Society (ERS)/Japanese Respiratory Society (JRS)/Latin American Thoracic Association (ALAT) guidelines for diagnosis of IPF, which describe diffuse mosaic attenuation/air trapping (bilateral, in three or more lobes) as being "inconsistent with UIP [usual interstitial pneumonia]" [4]. Furthermore, a recent update to the ATS/ERS/JRS/ALAT guidelines [5] and the recent Fleischner Society White Paper [6] describe "marked" or "extensive" mosaic attenuation as a finding "suggestive of an alternative diagnosis to IPF" or "consistent with a non IPF diagnosis", respectively, specifically hypersensitivity pneumonitis.

Despite the prominence in diagnostic guidelines of mosaic attenuation and air trapping as a CT feature suggestive of FHP, and inconsistent or atypical for IPF, these recommendations have not previously been validated, in particular with regard to their ability to distinguish IPF and FHP.

Furthermore, a number of questions arise as to how these CT signs should be used in clinical practice. First, several of the studies that have included mosaic attenuation in diagnostic models of hypersensitivity pneumonitis include non-FHP patients in their cohorts $[1,2]$, which may limit the applicability to patients with fibrosis.

Second, there is considerable variation in the use of terminology. Although mosaic attenuation is used as a single term in current guidelines [5, 6], mosaic attenuation consists of a group of different radiological entities, which may have variable diagnostic performance. Mosaic attenuation may be characterised by either lobules of low-attenuation lung or alternatively by patchy ground-glass opacity adjacent to preserved lobules of normal lung $[7,8]$. An additional term which has been referred to as the "headcheese sign" [9] represents the combination of preserved lobules and low-density lobules within the same lobe, and has recently been identified by an international survey of experts as being a commonly used CT feature of FHP [10]. Air trapping, meanwhile, is seen exclusively on expiratory CT and reflects low-density lobules seen at end-expiration (figure 1) [7]. The diagnostic utility of these various definitions of mosaic attenuation when applying diagnostic guidelines has also, to the best of our knowledge, not been previously assessed.

Therefore, the aims of our study were to 1) investigate the comparative frequency of mosaic attenuation in patients with FHP and IPF based on thresholds from published guidelines, and 2) interpret and validate the various thresholds of mosaic attenuation extent used in guidelines in distinguishing IPF and FHP. For both aims, we examined the impact of using differing definitions of mosaic attenuation, including air trapping identified on expiratory CT.

\section{Methods}

This retrospective study was approved by our institutional review board and patient consent was not required.
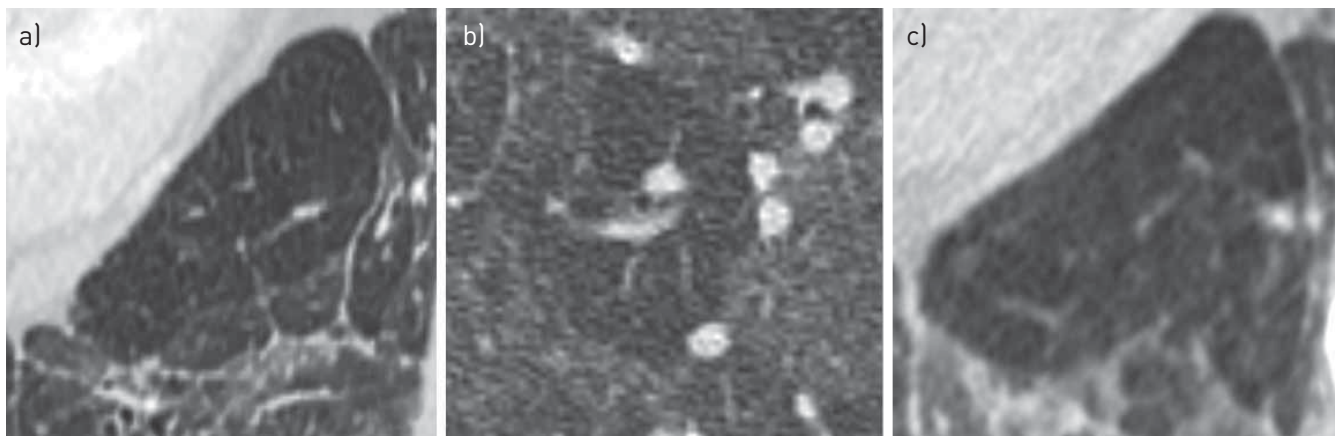

FIGURE 1 Mosaic attenuation on computed tomography (CT). a) Low-density lobules: the collection of lobules are of low density on this inspiratory CT relative to normal lung and contain constricted vessels. b) Preserved lobules: heterogeneous increased density of the surrounding lung; the central lobule is preserved. c) Air trapping: expiratory CT in the same patient as (a) accentuates this low density; the air-trapped lobule remains of similar density and volume. 


\section{Patient selection}

143 consecutive patients with a multidisciplinary team (MDT) diagnosis of FHP ( $n=64)$ or IPF ( $n=79)$ undergoing paired inspiratory and expiratory CT at our institution (Royal Brompton Hospital, London, UK) between September 2017 and September 2018 were retrospectively identified from a search of electronic patient records. Given previous studies questioning the utility of mosaic attenuation in distinguishing FHP and IPF [11], little weight is assigned to mosaic attenuation in our institution, when present as an isolated feature, to categorise CT scans as inconsistent with UIP. Nevertheless, to reduce the implicit radiological bias of using MDT diagnosis as the gold standard, case review was employed to identify "high-confidence" diagnoses of FHP and IPF (supplementary figure S1) requiring independent evidence of diagnosis that did not rely on mosaic attenuation on CT. Following the process of case review, 45 patients with FHP and 57 patients with IPF were included in the final derivation cohort.

For the validation cohort, an interstitial lung disease database of patients referred between 2010 and 2018 at a separate, secondary referral institution (Barnet Hospital, Royal Free NHS Foundation Trust, London, UK) was used to validate the CT findings, using identical definitions of high-confidence disease as in the derivation cohort (supplementary figure S2). The final validation cohort consisted of 62 patients (34 patients with IPF and 28 patients with FHP).

\section{CT protocol}

In both institutions CT scans were acquired with a 128-slice CT scanner (Somatom Sensation; Siemens, Erlangen, Germany). Inspiratory CT scans were performed at end-inspiration at $120 \mathrm{kV}$ with modulation of current, reference $100 \mathrm{mAs}$. Expiratory CT scans were performed at end-expiration, following patient training, at $120 \mathrm{kV}$ and $30 \mathrm{mAs}$, in the derivation cohort as a volume acquisition and in the validation cohort using interspaced imaging.

\section{CT analysis}

Mosaic attenuation

Mosaic attenuation was categorised and scored independently by two thoracic radiologists (A.D. and J.B.) blinded to the clinical data, using each of the following three definitions. 1) "Low-density lobules", defined as well-demarcated regions of low density containing constricted vessels (figure 1a). 2) "Preserved lobules", defined as well-demarcated lobules of normal lung surrounded by patchy ground-glass opacity (figure 1b). 3) "Air trapping" as assessed on expiratory CT, defined as well-demarcated lobules of low-density lung demonstrating a less than normal increase in attenuation, and lack of volume reduction, at expiration (figure 1c) [7].

Each mosaic attenuation variable was scored on a 7-point categorical scale (supplementary table S1) on a lobar basis, with the lingula defined as a sixth lobe. Post hoc combination of low-density lobules and preserved lobules present in the same lobe was considered representative of the headcheese sign (figure 2) [9].

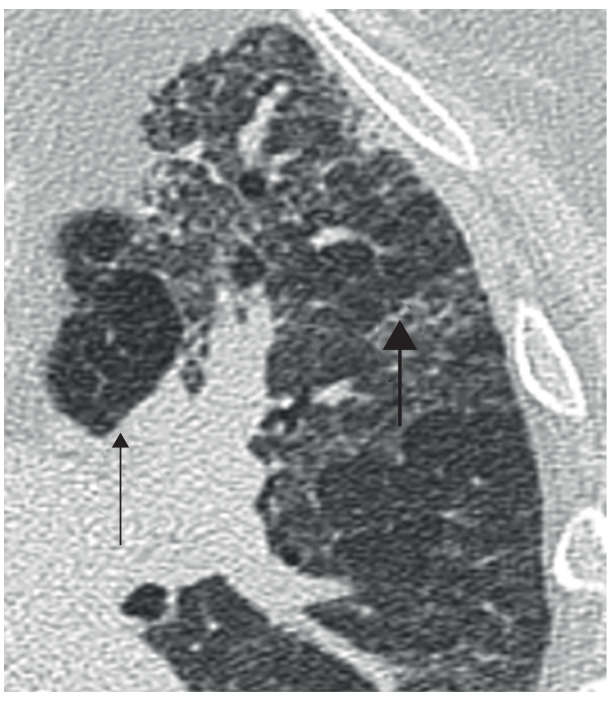

Patient 1

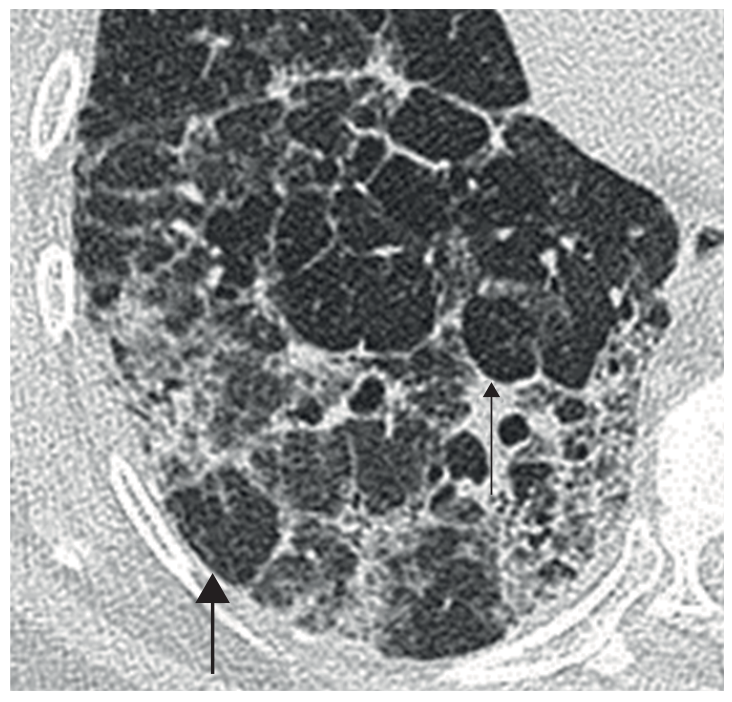

Patient 2

FIGURE 2 The headcheese sign in two different patients demonstrating differential lung density, comprising well-defined preserved lobules of normal lung (thick arrows) adjacent to ground-glass opacity and low-density lobules (thin arrows) in the same lobe, on inspiratory imaging. 
Consensing by agreement was performed in cases whenever there was disagreement between readers regarding the presence or absence of each variable on a lobar basis. For post hoc validation of mosaic attenuation scores against residual volume (RV)/total lung capacity (TLC) and maximal expiratory flow at $25-75 \%$ of forced vital capacity (MEF25-75\%) (see later), whole lung scores were calculated by summing the lobar low-density lobule scores for each patient.

\section{ATS/ERS/JRS/ALAT and Fleischner Society criteria for CT signs of mosaic attenuation and air trapping "inconsistent with UIP" or "suggestive of FHP as an alternative diagnosis to IPF"}

The 2011 ATS/ERS/JRS/ALAT guideline criteria state that the presence of mosaic attenuation/air trapping in three or more lobes bilaterally is "inconsistent with UIP" [4]. The 2017 Fleischner Society White Paper and the 2018 ATS/ERS/JRS/ALAT guideline update (referred to as "ATS/FS") do not state specific numerical values for mosaic attenuation that indicate FHP as an alternative diagnosis to IPF, but instead use the descriptors "extensive" [6] and "marked" [5]. Hence, we interpreted these terms as representing at least the same extent provided in the 2011 ATS/ERS/JRS/ALAT guideline guidance.

We therefore tested the following thresholds: 1) at least one lobule of mosaic attenuation in each of three lobes bilaterally (referred to as "2011 ATS criteria"), 2) three or more lobules of mosaic attenuation in each of three or more lobes bilaterally (referred to as "current ATS/FS criteria, $\geqslant 3$ lobules") and 3) five or more lobules of mosaic attenuation in each of three or more lobes bilaterally (referred to as "current ATS/FS criteria, $\geqslant 5$ lobules").

\section{Fibrosis and emphysema extent}

Fibrotic lung was defined by honeycomb cysts, reticulation or ground-glass opacity causing tractional airways dilatation. Both the fibrosis and emphysema extent were scored to the nearest $5 \%$ on a lobar basis by two thoracic radiologists (A.D. and J.B.). The mean reader score was calculated and then the mean of this score across all six lobes was taken as the whole lung score. The presence of a UIP pattern of fibrosis, mid/ upper lung predominance or peribronchovascularity was assessed by a separate radiologist (S.R.D.).

\section{Validation cohort}

In the validation cohort, CT data were analysed by two thoracic radiologists blinded to the clinical data (A.D. and a radiologist from an external institution (S.S.H.)) independently for signs of mosaic attenuation, at a threshold extrapolated from the derivation cohort in a binary manner. Fibrosis and emphysema extent were also scored using the derivation cohort methodology. The presence of a UIP pattern of fibrosis, mid/ upper lung predominance or peribronchovascularity was assessed by a separate radiologist (J.B.).

\section{Pulmonary function tests}

96 out of 102 patients (94.1\%) in the derivation cohort had pulmonary function test data within 3 months of CT imaging available from institutional records. Spirometry, diffusing capacity of the lung for carbon monoxide (DLCO) measurements and lung plethysmography were performed using established protocols, and values expressed as percentage predicted values [12]. 88 out of 96 patients ( $91.7 \%$ ) had MEF $25-75 \%$ measurements available; 73 out of 96 patients $(76.0 \%)$ were able to complete the flow-volume loop for estimation of RV/TLC.

\section{Statistical analysis}

Data and statistical analysis was performed in $\mathrm{R}$ (www.R-project.org). Interobserver agreement was measured using the interclass correlation coefficient for continuous variables and Cohen's $\kappa$ with quadratic weighting for categorical data. Comparisons of proportions of patients demonstrating mosaic attenuation at various guideline thresholds were made using the Chi-squared test for proportion or Fisher's exact test. The calculation of test statistics was made using the package epiR; confidence intervals for likelihood ratios were performed based on the methods of SIMEL et al. [13]. Linear models were constructed post hoc to assess the validity of visual scoring metrics against lung function parameters of air trapping and disease extent; independence of observations and homoscedasticity were confirmed using the Durban Watson test statistic, quantile-quantile plots and Breush-Pagan test.

\section{Results}

Patient demographics and observer agreement

The interclass correlation coefficient for fibrosis and emphysema extent in the derivation cohort was 0.84 and 0.92 , respectively. Cohen's $\kappa$ with quadratic ratings was good for low-density lobules and air trapping ( $\kappa=0.65$ and $\kappa=0.65$, respectively), and moderate for preserved lobules $(\kappa=0.48)$. 
Patient demographics are outlined in table 1. Patients with IPF were older, more likely to be male and with a greater pack-year smoking history. Patients with FHP had significantly more extensive fibrosis scored visually, with lower DLCO.

Frequency of mosaic attenuation in IPF and FHP according to "2011 ATS criteria" and "current ATS/FS criteria"

The frequency of patients meeting "2011 ATS criteria" and "current ATS/FS criteria" for FHP as an alternative diagnosis to IPF using different definitions of mosaic attenuation is demonstrated in table 2.

\section{Diagnostic utility of mosaic attenuation for diagnosis of FHP}

Table 3 demonstrates the test statistics for the various definitions and thresholds of mosaic attenuation. A positive test result is a patient meeting the threshold of FHP as an alternate diagnosis to IPF using varying thresholds and definitions for mosaic attenuation. A true positive test is a patient with a high-confidence (incorporating MDT diagnosis and case review) diagnosis of FHP.

The presence of the headcheese sign in three or more lobes, bilaterally, was highly specific (0.93) for a diagnosis of FHP and moderately sensitive (0.49). On the contrary, expiratory air trapping and its counterpart on inspiratory images, low-density lobules, were sensitive ( 0.82 and 0.78 , respectively) but only moderately specific for FHP (0.54 and 0.49 , respectively) when interpreted at the "2011 ATS criteria" thresholds.

Applying more stringent thresholds of mosaic attenuation to suggest FHP as an alternate diagnosis to IPF ("current ATS/FS criteria, $\geqslant 3$ lobules" and "current ATS/FS criteria, $\geqslant 5$ lobules") showed increased specificity of low-density lobules (0.86 and 0.96 , respectively) and expiratory air trapping (0.91 and 0.98 , respectively). These more stringent thresholds ("current ATS/FS criteria, $\geqslant 3$ lobules" and "current ATS/FS criteria, $\geqslant 5$ lobules") were associated with reduced sensitivity, relative to "2011 ATS criteria", for low-density lobules ( 0.51 and 0.16 , respectively) and expiratory air trapping ( 0.49 and 0.20 , respectively).

Validation of visual scoring metrics against lung function

To confirm that mosaic attenuation identified on inspiratory CT scans in this study represented physiological air trapping, post hoc linear models were created to examine the relationship of low-density lobule scores with pulmonary function indices of air trapping, i.e. RV/TLC $(n=73)$ and MEF25-75\% $(n=88)$. Low-density lobule scores were found to be significantly correlated with both air trapping indices, after adjustment for visual fibrosis and emphysema (tables 4 and 5). In order to assess for disease extent

\begin{tabular}{|c|c|c|c|}
\hline & IPF & FHP & p-value \\
\hline Patients & 57 & 45 & \\
\hline \multicolumn{4}{|l|}{ Demographics } \\
\hline Male & $48(84.2)$ & $18(40.0)$ & $<0.001$ \\
\hline Age years & $74.3 \pm 6.6$ & $63.4 \pm 9.2$ & $<0.001$ \\
\hline Ever-smoker & $39(68.4)$ & $18(40.0)$ & 0.005 \\
\hline Pack-year smoking history median (IQR) & $17(31.0)$ & 0 (12.5) & 0.005 \\
\hline \multicolumn{4}{|l|}{ Pulmonary function tests $\#$} \\
\hline DLCo & $44.6 \pm 12.7$ & $38.0 \pm 14.7$ & 0.02 \\
\hline FVC & $74.5 \pm 19.4$ & $67.6 \pm 23.4$ & 0.1 \\
\hline$M E F 25-75 \%$ ๆ & $82.0 \pm 38.6$ & $55.2 \pm 27.5$ & $<0.001$ \\
\hline $\mathrm{RV} / \mathrm{TLC}^{+}$ & $92.7 \pm 15.4$ & $115.0 \pm 27.9$ & $<0.001$ \\
\hline \multicolumn{4}{|l|}{ High-resolution CT } \\
\hline Fibrosis extent score & $19.0 \pm 9.2$ & $26.3 \pm 16.2$ & 0.009 \\
\hline Emphysema presence & $16(28.1)$ & $9(20.0)$ & 0.37 \\
\hline
\end{tabular}

Data are presented as $n, n(\%)$ or mean \pm SD, unless otherwise stated. IPF: idiopathic pulmonary fibrosis; FHP: fibrotic hypersensitivity pneumonitis; IQR: interquartile range; DLCO: diffusing capacity of the lung for carbon monoxide; MEF25-75\%: maximal expiratory flow at $25-75 \%$ of forced vital capacity; RV: residual volume; TLC: total lung capacity; CT: computed tomography. ${ }^{*}$ : available in 96 patients within 3 months of scan (IPF $n=53$; FHP $n=43$ ); " : eight patients with IPF were unable to perform MEF25-75\% measurements: ${ }^{+}: 11$ patients with FHP and 12 patients with IPF were unable to perform RV/TLC measurements. Data were compared using Fischer's exact test (sex, smoking status and emphysema presence), the t-test lage, pulmonary function tests and fibrosis extent) or the Mann-Whitney test (pack-year history). $p<0.05$ was considered significant. 
TABLE 2 Frequency of computed tomography signs of mosaic attenuation present according to "2011 ATS criteria"\# or "current ATS/FS criteria" " thresholds for fibrotic hypersensitivity pneumonitis (FHP) as an alternative diagnosis to idiopathic pulmonary fibrosis (IPF)

\begin{tabular}{lccc} 
& IPF & FHP & p-value \\
\hline Patients & 57 & 45 & \\
Preserved lobules & & & \\
$\quad$ 2011 ATS criteria & $11(19.3)$ & $27(60.0)$ & $<0.001$ \\
$\quad$ Current ATS/FS criteria, $\geqslant 3$ lobules & $4(7.0)$ & $15(33.3)$ & $<0.001$ \\
$\quad$ Current ATS/FS criteria, $\geqslant 5$ lobules & $1(1.8)$ & $8(17.8)$ & 0.01 \\
Low-density lobules & $29(50.9)$ & $35(77.8)$ & 0.005 \\
2011 ATS criteria & $8(14.0)$ & $23(51.1)$ & $<0.001$ \\
$\quad$ Current ATS/FS criteria, $\geqslant 3$ lobules & $2(3.5)$ & $7(15.6)$ & 0.04 \\
$\quad$ Current ATS/FS criteria, $\geqslant 5$ lobules & $4(7.0)$ & $22(48.9)$ & $<0.001$ \\
Headcheese sign & $4(7.0)$ & $22(48.9)$ & $<0.001$ \\
$\quad 2011$ ATS criteria & $2(3.5)$ & $19(38.8)$ & $<0.001$ \\
$\quad$ Current ATS/FS criteria, $\geqslant 3$ lobules & & \\
$\quad$ Current ATS/FS criteria, $\geqslant 5$ lobules & $26(45.6)$ & $37(82.2)$ & $<0.001$ \\
Expiratory air trapping & $5(8.8)$ & $22(48.9)$ & $<0.001$ \\
$\quad$ 2011 ATS criteria & $1(1.8)$ & $9(20.0)$ & 0.004 \\
$\quad$ Current ATS/FS criteria, $\geqslant 3$ lobules & & \\
$\quad$ Current ATS/FS criteria, $\geqslant 5$ lobules & & \\
\hline
\end{tabular}

Data are presented as $\mathrm{n}$ or $\mathrm{n}$ (\%), unless otherwise stated. \#: 2011 American Thoracic Society (ATS)/ European Respiratory Society (ERS)/Japanese Respiratory Society (JRS)/Latin American Thoracic Association (ALAT) guidelines [4]; ๆ: 2018 ATS/ERS/JRS/ALAT guidelines [5]/2017 Fleischner Society White Paper [6]. Frequency of various computed tomography signs of mosaic attenuation by diagnosis, using different definitions of mosaic attenuation and different thresholds of mosaic attenuation for FHP based on guidelines. "2011 ATS criteria" requires at least one lobule in three or more lobes, bilaterally. "Current ATS/FS criteria, $\geqslant 3$ lobules" requires three or more lobules in three or more lobes bilaterally. "Current ATS/FS criteria, $\geqslant 5$ lobules" requires five or more lobules in three or more lobes bilaterally. Headcheese represents the sum of both low-density lobules and preserved lobules only when both are present in the same lobe. $p<0.05$ was considered significant.

confounding our visual scores of air trapping, a separate linear model found that both visual fibrosis and emphysema scores were correlated with DLCO; however, visual low-density lobule score was not (table 6).

\section{Validation of findings against an external cohort}

In order to validate our findings, the threshold of "three of more lobules, in three or more lobes, bilaterally" was selected as the optimal balance between high specificity and acceptable sensitivity for a diagnosis of FHP, as an alternate diagnosis to IPF. This threshold was applied to CT data from an external cohort, derived from a database of interstitial lung disease at a secondary referral institution for interstitial lung disease (supplementary figure S2). Data is presented for each reader; consensing was not performed. Agreement between the two scorers in the validation cohort was substantial for low-density lobules and the headcheese sign $(\kappa=0.64$ and $\kappa=0.71$, respectively), and moderate for expiratory air trapping $(\kappa=0.59)$. As in the derivation cohort, patients with IPF were older, more frequently male, more frequently ever-smokers and had a greater pack-year smoking history (table 7).

Test statistics for the validation cohort are displayed in table 8 . The headcheese sign retained its high specificity for both readers ( 0.94 and 0.95 , respectively), whereas the sensitivity was greater than that seen in the derivation cohort ( 0.71 and 0.64 , respectively). The specificity of both low-density lobules $(0.65$ and 0.65 , respectively) and expiratory air trapping ( 0.74 and 0.65 , respectively) was, however, decreased relative to the derivation cohort.

\section{Analysis of the headcheese sign against other CT features of FHP}

In order to analyse the utility of the headcheese sign as an isolated feature, CT data from the derivation and validation cohort were combined and assessed post hoc for the presence of other features of FHP, i.e. upper lobe predominance and/or a peribronchovascular distribution of fibrosis, sparing the subpleural lung [6]. A total of 37 out of 73 patients had at least one of these signs present (eight out of 73 showed upper/mid lobe predominance in isolation, 10 out of 73 showed peribronchovascularity in isolation and 19 out of 73 showed both signs). 
TABLE 3 Test statistics for "2011 ATS criteria"\# and extrapolated "current ATS/FS criteria"ף thresholds for fibrotic hypersensitivity pneumonitis (FHP) as an alternative diagnosis to idiopathic pulmonary fibrosis (IPF)

Sensitivity Specificity PPV NPV Positive likelihood
ratio $(95 \% \mathrm{CI})$

Negative likelihood ratio $(95 \% \mathrm{Cl})$

\begin{tabular}{|c|c|c|c|c|c|c|}
\hline \multicolumn{7}{|l|}{ Preserved lobules } \\
\hline 2011 ATS criteria & 0.60 & 0.81 & 0.71 & 0.72 & $3.11(1.74-5.56)$ & $0.50(0.34-0.72)$ \\
\hline Current ATS/FS criteria, $\geqslant 3$ lobules & 0.33 & 0.93 & 0.79 & 0.64 & $4.75(1.69-13.32)$ & $0.72(0.58-0.89)$ \\
\hline Current ATS/FS criteria, $\geqslant 5$ lobules & 0.18 & 0.98 & 0.89 & 0.60 & $10.13(1.32-78.07)$ & $0.84(0.73-0.96)$ \\
\hline \multicolumn{7}{|l|}{ Low-density lobules } \\
\hline 2011 ATS criteria & 0.78 & 0.49 & 0.55 & 0.74 & $1.53(1.13-2.06)$ & $0.45(0.25-0.83)$ \\
\hline Current ATS/FS criteria, $\geqslant 3$ lobules & 0.51 & 0.86 & 0.74 & 0.69 & $3.64(1.80-7.36)$ & $0.57(0.41-0.78)$ \\
\hline Current ATS/FS criteria, $\geqslant 5$ lobules & 0.16 & 0.96 & 0.78 & 0.59 & $4.43(0.97-20.31)$ & $0.88(0.76-1.00)$ \\
\hline \multicolumn{7}{|l|}{ Headcheese } \\
\hline 2011 ATS criteria & 0.49 & 0.93 & 0.85 & 0.7 & $6.97(2.59-18.77)$ & $0.55(0.41-0.74)$ \\
\hline Current ATS/FS criteria, $\geqslant 3$ lobules & 0.49 & 0.93 & 0.85 & 0.7 & $6.97(2.59-18.77)$ & $0.55(0.41-0.74)$ \\
\hline Current ATS/FS criteria, $\geqslant 5$ lobules & 0.42 & 0.96 & 0.90 & 0.68 & $12.03(2.96-48.97)$ & $0.60(0.46-0.77)$ \\
\hline \multicolumn{7}{|l|}{ Expiratory air trapping } \\
\hline 2011 ATS criteria & 0.82 & 0.54 & 0.59 & 0.79 & $1.8(1.32-2.47)$ & $0.33(0.17-0.64)$ \\
\hline Current ATS/FS criteria, $\geqslant 3$ lobules & 0.49 & 0.91 & 0.81 & 0.69 & $5.57(2.29-13.56)$ & $0.56(0.42-0.75)$ \\
\hline Current ATS/FS criteria, $\geqslant 5$ lobules & 0.20 & 0.98 & 0.90 & 0.61 & $11.40(1.50-86.69)$ & $0.81(0.70-0.95)$ \\
\hline
\end{tabular}

PPV: positive predictive value; NPV: negative predictive value. " 2011 American Thoracic Society (ATS)/European Respiratory Society (ERS)/ Japanese Respiratory Society (JRS)/Latin American Thoracic Association (ALAT) guidelines [4]; ": 2018 ATS/ERS/JRS/ALAT guidelines [5]/2017 Fleischner Society White Paper [6]. Meeting the threshold for FHP as an alternate diagnosis to IPF is denoted as a positive test result. A true positive is a patient with high-confidence FHP.

In patients with FHP, the headcheese sign was not shown to segregate with other CT features of FHP (Fisher's test, $\mathrm{p}=0.81$ ). When patients with other CT features of FHP were excluded from the analysis, the headcheese sign retained high specificity (0.93) and moderate sensitivity (0.56) for a diagnosis of high-confidence FHP, as opposed to IPF.

\section{Discussion}

In our study, we have attempted to validate current diagnostic guidelines that are in widespread use by radiologists and pulmonologists when attempting to differentiate FHP from IPF on CT. In keeping with prior studies $[3,11]$, we have shown that mosaic attenuation is a common finding in IPF, being present in up to $51 \%$ of patients diagnosed according to the "2011 ATS criteria". This suggests that mosaic attenuation should not be considered a feature that is necessarily inconsistent with, or atypical for, IPF.

This point is reinforced when evaluating the guidance described in current diagnostic guideline updates. In the absence of a specific definition of the terms "marked" [5] and "extensive" [6], we chose to extrapolate the "2011 ATS criteria" as these have a specific, reproducible threshold. We chose two interpretations of varying extent; requiring three or more lobules and five or more lobules of each mosaic attenuation variable, in three or more lobes, bilaterally. We found that although increasing the required extent of mosaic attenuation on CT to suggest FHP as an alternative diagnosis to IPF improved diagnostic specificity, even at the most extensive extrapolation of the Fleischner Society White Paper [6] and 2018 ATS/ERS/JRS/ALAT clinical practice guidelines [5] (requiring five lobules of a given mosaic attenuation variable within three or more lobes, bilaterally), no threshold of mosaic attenuation could completely exclude a diagnosis of IPF. Furthermore, such a profuse extent of mosaic attenuation was associated with poor sensitivity, whether defined by preserved lobules, low-density lobules or expiratory air trapping, thus potentially limiting the clinical utility of these CT features.

The "headcheese sign", first described by WEBB et al. [9], is common parlance among radiologists and refers to a specific form of mosaic attenuation, describing the combination of ground-glass opacity, preserved lobules and low-attenuation lobules. Indeed, in a recent Delphi survey [10], this sign reached consensus as important in the diagnosis of FHP. The headcheese sign has, however, not been previously examined with regard to its diagnostic utility. We found the presence of the headcheese sign to be strongly specific for a diagnosis of FHP, outperforming all other mosaic attenuation metrics, and thus out of all the mosaic attenuation variables can be considered atypical for IPF and more suggestive of FHP, at any threshold. Indeed, the headcheese sign retained high specificity when examined in an external cohort of patients with IPF and FHP, and also when analysed in a subgroup where other CT signs of FHP were excluded. 
TABLE 4 Linear model of residual volume (RV)/total lung capacity (TLC) against visual low-density lobule score, visual fibrosis extent and visual emphysema extent

\begin{tabular}{lcc} 
& $\boldsymbol{\beta}$-estimate \pm SE & p-value \\
\hline Low-density lobules & $2.01 \pm 0.42$ & $<0.001$ \\
Fibrosis & $0.28 \pm 0.20$ & 0.16 \\
Emphysema & $0.09 \pm 0.25$ & 0.73 \\
\hline
\end{tabular}

Includes 73 patients with RV/TLC available. Adjusted $R^{2}=0.29, p<0.001 . p<0.05$ was considered significant.

\begin{tabular}{|c|c|c|}
\hline & $\beta$-estimate $\pm \mathrm{SE}$ & p-value \\
\hline Low-density lobules & $-2.54 \pm 0.64$ & $<0.001$ \\
\hline Fibrosis & $-0.13 \pm 0.28$ & 0.66 \\
\hline Emphysema & $-0.77 \pm 0.41$ & 0.06 \\
\hline
\end{tabular}

Includes 88 patients with MEF25-75\% available. Adjusted $R^{2}=0.15, p<0.001 . p<0.05$ was considered significant.

TABLE 6 Linear model of diffusing capacity of the lung for carbon monoxide ( $D$ LCO) against visual low-density lobule score, visual fibrosis extent and visual emphysema extent

\begin{tabular}{lcc} 
& $\boldsymbol{\beta}$-estimate \pm SE & p-value \\
\hline Low-density lobules & $-0.36 \pm 0.22$ & 0.1 \\
Fibrosis & $-0.56 \pm 0.09$ & $<0.001$ \\
Emphysema & $-0.46 \pm 0.13$ & $<0.001$ \\
\hline
\end{tabular}

Includes 96 patients with $D L C 0$ available. Adjusted $R^{2}=0.33, p<0.001 . p<0.05$ was considered significant.

Our findings show that the headcheese sign co-segregates with high-confidence clinical features of FHP and is rarely associated with high-confidence clinical variables of IPF. While it would not be robust to extrapolate the utility of the headcheese sign to low-confidence or unclassifiable diagnoses (which were not examined in this cohort), the headcheese sign can be regarded as significant when the MDT is faced with incomplete clinical data. Furthermore, the headcheese sign is widely known among radiologists and has the potential to be a distinctive, widely useable CT feature suggestive of FHP as an alternative diagnosis to IPF.

When applied to the validation cohort, the high specificity of the "current ATS/FS criteria, $\geqslant 3$ lobules" threshold was not maintained for low-density lobules or air trapping. This combination of decreased specificity and increased sensitivity may reflect increased incidence of air trapping in this population. The variability of these findings further limits the confidence of low-density lobules and expiratory air trapping at the thresholds examined, when seen in the absence of the headcheese sign.

Mosaic attenuation has previously been described as an important CT parameter in diagnostic models of hypersensitivity pneumonitis [1,2]. However, one important distinction which should be made between this study and previous diagnostic models of hypersensitivity pneumonitis $[1,2]$ is that our cohort contains only patients with FHP (as opposed to nonfibrotic disease). Patients with nonfibrotic disease, by definition, fall within a different diagnostic conundrum where IPF is not a valid differential diagnosis. The applicability of diagnostic models derived from partly nonfibrotic cohorts to distinguish FHP from IPF is uncertain.

The rationale for employing CT features of mosaic attenuation as a sign of FHP is based, in part, on the airways centric fibrosis demonstrated on histopathology. While a UIP histology lacks implicit airways disease, a major confounder is smoking history, which is often encountered in patients with IPF [14]. A previous radiological study in 250 healthy subjects found $27 \%$ of ex-smokers demonstrated expiratory air 


\begin{tabular}{|c|c|c|c|}
\hline Characteristic & IPF & FHP & p-value \\
\hline Patients & 34 & 28 & \\
\hline \multicolumn{4}{|l|}{ Demographics } \\
\hline Male & 30 (88.2) & $13(46.4)$ & 0.001 \\
\hline Age years & $78.0 \pm 7.4$ & $70.0 \pm 11.2$ & 0.002 \\
\hline Ever-smoker & 22 (64.7) & $9(32.1)$ & 0.02 \\
\hline Pack-year smoking history median (IQR) & 20 (35) & $0(3)$ & 0.002 \\
\hline \multicolumn{4}{|l|}{ High-resolution CT } \\
\hline Fibrosis extent score & $32.9 \pm 13.0$ & $23.2 \pm 14.4$ & 0.007 \\
\hline Emphysema presence & $7(20.6)$ & $4(14.3)$ & 0.7 \\
\hline \multicolumn{4}{|c|}{$\begin{array}{l}\text { Data are presented as } n, n(\%) \text { or mean } \pm S D \text {, unless otherwise stated. IPF: idiopathic pulmonary fibrosis; } \\
\text { FHP: fibrotic hypersensitivity pneumonitis; IQR: interquartile range; CT: computed tomography. Data were } \\
\text { compared using Fischer's exact test (sex, smoking status and emphysema presence), the t-test lage and } \\
\text { fibrosis extent) or the Mann-Whitney test (pack-year history). } p<0.05 \text { was considered significant. }\end{array}$} \\
\hline
\end{tabular}

trapping of segmental or lobar extent, a significantly more frequent finding than in never-smokers [15]. This may explain the high frequency of mosaic attenuation and air trapping in patients with IPF in our and previous studies [11].

The examination of visual scores against RV/TLC, MEF25-75\% and DLCO was undertaken to examine the reliability of our visual scores of air trapping, and the possible confounding of emphysema on both visual scores and pulmonary function tests of small airways disease. Emphysema affects both measures of airflow and DLCO, whereas intrinsic airways disease should not influence DLCO [16], unless severe. The association of our visual scores of air trapping with both airflow metrics, and their independence from DLCO, suggests our visual scores are robust.

One limitation to our study is the reliance of MDT diagnosis to establish gold standard diagnosis. Radiologist interpretation of CT images forms a key part of the MDT reaching a diagnosis, potentially confounding any study on CT findings. We believe our methodology is robust for two reasons. First, since the findings of the IPFnet study [11], we have given little weight to low-density lobules suggestive of physiological air trapping in our MDT, especially when present in a patient who would be otherwise of "definite" or "probable" UIP morphology. Second, our case review ensured "high-confidence" diagnoses only were included, excluding cases based solely on radiological findings. While the criteria used for this case review have previously been used elsewhere [2], in the diagnosis of hypersensitivity pneumonitis, the lack of standardised international criteria for diagnosis of FHP adds heterogeneity to this cohort. Our institution acts as a tertiary referral centre for interstitial lung disease and this may introduce patient selection bias towards more severe disease. However, our findings with respect to the headcheese sign were also applicable in a secondary referral centre population.

TABLE 8 Test statistics for visual scores of different mosaic attenuation variables in the validation cohort

\begin{tabular}{|c|c|c|c|c|}
\hline & \multicolumn{2}{|c|}{ Reader 1} & \multicolumn{2}{|c|}{ Reader 2} \\
\hline & Sensitivity & Specificity & Sensitivity & Specificity \\
\hline \multicolumn{5}{|l|}{ Low-density lobules } \\
\hline Current ATS/FS criteria, $\geqslant 3$ lobules & 0.75 & 0.65 & 0.79 & 0.65 \\
\hline \multicolumn{5}{|l|}{ Headcheese sign } \\
\hline Current ATS/FS criteria, $\geqslant 3$ lobules & 0.71 & 0.94 & 0.64 & 0.95 \\
\hline \multicolumn{5}{|l|}{ Expiratory air trapping } \\
\hline Current ATS/FS criteria, $\geqslant 3$ lobules & 0.64 & 0.74 & 0.79 & 0.65 \\
\hline \multicolumn{5}{|c|}{$\begin{array}{l}\text { Test statistics for the mosaic attenuation variables using the "three or more lobules, in three or more } \\
\text { lobes, bilaterally" threshold extrapolated from the } 2018 \text { American Thoracic Society/European Respiratory } \\
\text { Society/Japanese Respiratory Society/Latin American Thoracic Association guidelines [5]/2017 Fleischner } \\
\text { Society White Paper [6] ("current ATS/FS criteria, } \geqslant 3 \text { lobules"). Meeting the threshold for fibrotic } \\
\text { hypersensitivity pneumonitis (FHP) as an alternate diagnosis to idiopathic pulmonary fibrosis is denoted as } \\
\text { a positive test result. A true positive is a patient with FHP. }\end{array}$} \\
\hline
\end{tabular}


Another limitation of this study is the potential for CT scorers to have unconscious biases towards scoring of mosaic attenuation, based on ancillary CT features of FHP. The independence of the headcheese sign from other CT features of FHP, and the retained high specificity when patients with ancillary CT features of FHP were excluded, suggests that this effect was minimal.

In conclusion, we have shown that the individual components of the heterogeneous term of "mosaic attenuation" have variable diagnostic performance and therefore caution should be applied when referring to the term "mosaic attenuation" without clarification. Thresholds for "extensive" or "marked" mosaic attenuation based on current guidelines are associated with improved specificity but reduced sensitivity for FHP. The headcheese sign, however, should be confidently considered a CT sign of FHP as an alternate diagnosis to IPF.

Conflict of interest: J. Barnett has nothing to disclose. P.L. Molyneaux has, via his institution, received industry academic funding from Roche, Boehringer Ingelheim and Galapagos, and has received lecture fees from Roche. B. Rawal has nothing to disclose. R. Abdullah has nothing to disclose. S.S. Hare has nothing to disclose. R. Vancheeswaran has nothing to disclose. S.R. Desai is co-organiser of an educational course convened by Boehringer Ingelheim. This is a "hands-on" workshop for pulmonologists and radiologists working in the field. The aim of the course is purely educational and does not focus on any particular disease (and specifically not IPF or hypersensitivity pneumonitis), nor does it promote any specific treatment or management. T.M. Maher has, via his institution, received industry academic funding from GlaxoSmithKline R\&D and UCB, and has received consultancy or lecture fees from Apellis, AstraZeneca, aTyr Pharma, Bayer, Biogen Idec, Boehringer Ingelheim, Galapagos, GlaxoSmithKline R\&D, Indalo, Pliant, ProMetic, Roche, Samumed and UCB, and has stock options in Apellis. A.U. Wells reports personal fees from Intermune, Boehringer Ingelheim, Gilead, MSD, Roche, Bayer and Chiesi, outside the submitted work. A. Devaraj reports personal fees from GlaxoSmithKline, Roche and Boehringer Ingelheim, outside the submitted work.

\section{References}

1 Johannson KA, Elicker BM, Vittinghoff E, et al. A diagnostic model for chronic hypersensitivity pneumonitis. Thorax 2016; 71: 951-954.

2 Salisbury ML, Gross BH, Chughtai A, et al. Development and validation of a radiological diagnosis model for hypersensitivity pneumonitis. Eur Respir J 2018; 52: 1800443.

3 Silva CIS, Muller NL, Lynch DA, et al. Chronic hypersensitivity pneumonitis: differentiation from idiopathic pulmonary fibrosis and nonspecific interstitial pneumonia by using thin-section CT. Radiology 2008; 246: 288-297.

4 Raghu G, Collard HR, Egan JJ, et al. An official ATS/ERS/JRS/ALAT statement: idiopathic pulmonary fibrosis: evidence-based guidelines for diagnosis and management. Am J Respir Crit Care Med 2011; 183: 788-824.

5 Raghu G, Remy-Jardin M, Myers JL, et al. Diagnosis of Idiopathic Pulmonary Fibrosis. An Official ATS/ERS/JRS/ ALAT Clinical Practice Guideline. Am J Respir Crit Care Med 2018; 198: e44-e68.

6 Lynch DA, Sverzellati N, Travis WD, et al. Diagnostic criteria for idiopathic pulmonary fibrosis: a Fleischner Society White Paper. Lancet Respir Med 2018; 6: 138-153.

7 Hansell DM, Bankier AA, MacMahon H, et al. Fleischner Society: glossary of terms for thoracic imaging. Radiology 2008; 246: 697-722.

8 Stern EJ, Swensen SJ, Hartman TE, et al. CT mosaic pattern of lung attenuation: distinguishing different causes. Am J Roentgenol 1995; 165: 813-816.

9 Webb WR, Wayne R, Müller NL. High-Resolution CT of the Lung. Philadelphia, Wolters Kluwer, 2009.

10 Morisset J, Johannson KA, Jones KD, et al. Identification of diagnostic criteria for chronic hypersensitivity pneumonitis: an international modified Delphi survey. Am J Respir Crit Care Med 2018; 197: 1036-1044.

11 Yagihashi K, Huckleberry J, Colby TV, et al. Radiologic-pathologic discordance in biopsy-proven usual interstitial pneumonia. Eur Respir J 2016; 47: 1189-1197.

12 Quanjer PH, Tammeling GJ, Cotes JE, et al. Lung volumes and forced ventilatory flows. Eur Respir J 1993; 6: 5-40.

13 Simel DL, Samsa GP, Matchar DB. Likelihood ratios with confidence: sample size estimation for diagnostic test studies. J Clin Epidemiol 1991; 44: 763-770.

14 Baumgartner KB, Samet JM, Stidley CA, et al. Cigarette smoking: a risk factor for idiopathic pulmonary fibrosis. Am J Respir Crit Care Med 1997; 155: 242-248.

15 Mastora I, Remy-Jardin M, Sobaszek A, et al. Thin-section CT finding in 250 volunteers: assessment of the relationship of CT findings with smoking history and pulmonary function test results. Radiology 2001; 218: 695-702.

16 Hansell DM, Rubens MB, Padley SP, et al. Obliterative bronchiolitis: individual CT signs of small airways disease and functional correlation. Radiology 1997; 203: 721-726. 